\title{
Page curve from dynamical branes in JT gravity
}

\author{
Kazumi Okuyama $^{a}$ and Kazuhiro Sakai ${ }^{b}$ \\ ${ }^{a}$ Department of Physics, Shinshu University, \\ 3-1-1 Asahi, Matsumoto 390-8621, Japan \\ ${ }^{b}$ Institute of Physics, Meiji Gakuin University, \\ 1518 Kamikurata-cho, Totsuka-ku, Yokohama 244-8539, Japan \\ E-mail: kazumi@azusa.shinshu-u.ac.jp, kzhrsakai@gmail.com
}

ABSTRACT: We study the Page curve of an evaporating black hole using a toy model given by Jackiw-Teitelboim gravity with Fateev-Zamolodchikov-Zamolodchikov-Teschner (FZZT) antibranes. We treat the anti-FZZT branes as dynamical objects, taking their back-reaction into account. We construct the entanglement entropy from the dual matrix model and study its behavior as a function of the 't Hooft coupling $t$ proportional to the number of branes, which plays the role of time. By numerical computation we observe that the entropy first increases and then decreases as $t$ grows, reproducing the well-known behavior of the Page curve of an evaporating black hole. The system finally exhibits a phase transition, which may be viewed as the end of the evaporation. We study the critical behavior of the entropy near the phase transition. We also make a conjecture about the late-time monotonically decreasing behavior of the entropy. We prove it in a certain limit as well as give an intuitive explanation by means of the dual matrix model.

KeYwords: 2D Gravity, Black Holes, Matrix Models

ArXiv EPrint: 2111.09551 


\section{Contents}

1 Introduction 1

2 Entropy of radiation from dynamical anti-FZZT branes 3

2.1 Matrix integral and black hole microstates 3

2.2 Ensemble average 4

2.3 Reduced density matrix of radiation $\quad 5$

2.4 Resolvent of reduced density matrix and entropy 8

3 Phase transition $\quad 10$

$\begin{array}{lll}3.1 & \text { Threshold energy } & 10\end{array}$

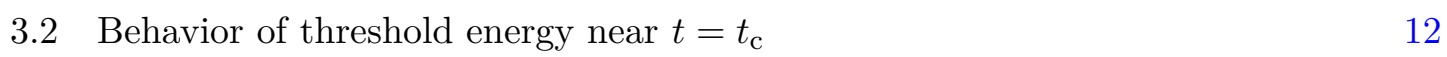

3.3 Effective zero-temperature entropy and von Neumann entropy near $t=t_{\mathrm{c}} \quad 12$

4 Numerical study of Page curve 13

$\begin{array}{ll}4.1 \text { von Neumann entropy } & 13\end{array}$

$\begin{array}{ll}4.2 \text { Rényi entropy } & 13\end{array}$

$5 \quad$ Monotonicity of $\widetilde{\boldsymbol{S}}_{\boldsymbol{n}} \quad \mathbf{1 6}$

$\begin{array}{lll}5.1 & \text { Leading-order eigenvalue density and its derivative } & 16\end{array}$

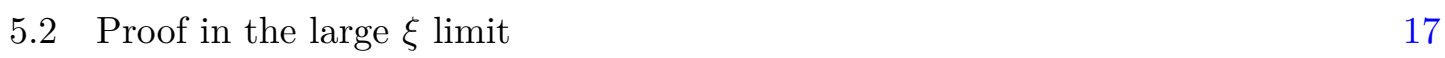

$\begin{array}{ll}\text { 5.3 Intuitive understanding of the decreasing behavior of } \widetilde{S}_{n} & 19\end{array}$

$\begin{array}{llr}6 & \text { Dilaton gravity } & 21\end{array}$

$\begin{array}{lll}7 & \text { Conclusion and outlook } & 23\end{array}$

A Schwinger-Dyson equation from saddle point approximation $\quad 24$

\section{Introduction}

The black hole information paradox has been a long-standing puzzle in the study of quantum gravity [1]. In particular, the growing behavior of the entropy of thermal radiation based on Hawking's calculation [2] apparently contradicts with the unitarity of the quantum mechanics which requires that the black hole stays in a pure state. For an evaporating black hole, the Page curve [3], a plot of the entanglement entropy of the Hawking radiation as a function of time, should show a decreasing behavior toward the end of evaporation. Recent studies revealed that the gravitational path integral receives, even semi-classically, contributions from saddle-points other than the classical black hole solution, namely the replica wormholes $[4,5]$. This is a key to understand how the Page curve is obtained in an 
expected form, which partly resolves the information paradox. The idea was refined in the form of the island formula [6], which was first derived by means of holography [4-12] and then consolidated by directly evaluating the gravitational path integral in quantum gravity in two dimensions $[13,14]$. See [15] for a recent review and references therein.

In [13] the Page curve was studied by using Jackiw-Teitelboim (JT) gravity $[16,17]$ with the end-of-the-world (EOW) branes. ${ }^{1}$ Roughly speaking, the system is viewed as a generalization of the original Page's model [19] (see [20] for recent exact results). Page's calculation starts with a random pure state in the bipartite Hilbert space consisting of two subspaces that represent the interior and exterior of a black hole. Taking ensemble average of the state in either of the subspaces, one obtains the reduced density matrix, from which the entanglement entropy is calculated. In Page's model the ensemble is Gaussian in both subspaces. In the case of JT gravity with EOW branes, the ensemble in the interior is Gaussian whereas the average in the exterior is described by the double-scaled matrix integral of JT gravity [21]. The size of the interior subspace, which is identified with the number of branes, plays the role of time.

In this paper we propose another simple toy model to understand the Page curve: JT gravity with Fateev-Zamolodchikov-Zamolodchikov-Teschner (FZZT) antibranes [22, 23]. Our model is a simplified variant of the model of [13], with the EOW branes replaced by anti-FZZT branes. In our previous paper [24] we showed that the matrix model description of the EOW brane in [25] corresponds to that of a collection of infinitely many anti-FZZT branes with a particular set of parameters. It is therefore simpler to consider JT gravity with a single kind of anti-FZZT branes.

Despite this simplification, our model captures several features of black hole entropy. Most notably, the entanglement entropy exhibits the late-time decreasing behavior which is characteristic of an evaporating black hole. ${ }^{2}$ To reproduce this decreasing behavior, it is crucial to treat branes as dynamical objects. In the previous studies, branes are treated as either dynamical [25] or non-dynamical [13]. We will see from numerical computation that the late-time decreasing behavior of the entropy is reproduced only when we treat antiFZZT branes as dynamical objects. In fact, we consider the 't Hooft limit, in which the back-reaction of branes is not negligible and one has to treat branes as dynamical objects. We will also study how this decreasing behavior arises from the viewpoint of the matrix model and make a conjecture about monotonicity, which we will prove in a certain limit.

Our model exhibits a phase transition as "time" grows. The transition may be viewed as the end of the evaporation of black hole. We will study the critical behavior of the entropy near the transition point. One can consider JT gravity with FZZT branes and study the Page curve in the same manner. In this case, however, no phase transition occurs and the entropy continue increasing. All these results derived from the matrix model are in perfect accordance with the semi-classical analysis on the gravity side: Dilaton gravities with nontrivial dilaton potential were studied as deformations of JT gravity [28, 29] and black hole solutions in these gravities were also studied [30]. JT gravity with (anti-)FZZT

\footnotetext{
${ }^{1}$ A classification of branes in JT gravity is found in [18].

${ }^{2}$ The Page curve of an evaporating black hole in JT gravity was studied in different approaches [26, 27].
} 
branes can be viewed as this type of dilaton gravity [24]. We will study its black hole solutions and see the continuous growth of the entropy in the FZZT setup and the phase transition in the anti-FZZT setup. Thus, in this paper we concentrate on the case of anti-FZZT branes.

This paper is organized as follows. In section 2, we describe our model and explain the general method of computing the entropy of the Hawking radiation. In section 3, we explain how the phase transition occurs and study the critical behavior of the entropy. In section 4 , we numerically study the Page curve, i.e. the evolution of the entropy as a function of the 't Hooft coupling. We also make a conjecture about the late-time monotonically decreasing behavior of the entropy. In section 5 , we prove the conjecture in a certain limit. We also give an intuitive explanation of the reason why the entropy decreases. In section 6 , we study black hole solutions from the viewpoint of dilaton gravity. Finally we conclude in section 7. In appendix A, we give a derivation of the Schwinger-Dyson equation (2.22) based on the saddle point method.

\section{Entropy of radiation from dynamical anti-FZZT branes}

In this section we will describe our model and explain the general method of computing the entropy of the Hawking radiation. In many parts of our formulation we follow the method of [13] with EOW branes being replaced by anti-FZZT branes. In our study, however, branes are treated as dynamical objects. This is along the lines of [25] and an important difference from [13].

\subsection{Matrix integral and black hole microstates}

Let us consider general $2 \mathrm{~d}$ topological gravity with $K$ dynamical anti-FZZT branes. ${ }^{3}$ It is described by the double scaling limit of the matrix integral

$$
\begin{aligned}
Z & =\int d H e^{-\operatorname{Tr} V(H)} \operatorname{det}(\xi+H)^{-K} \\
& =\int d H d Q d Q^{\dagger} e^{-\operatorname{Tr} V(H)-\operatorname{Tr} Q^{\dagger}(\xi+H) Q}
\end{aligned}
$$

Here $H$ and $Q$ are $N \times N$ hermitian and $N \times K$ complex matrices respectively. $\xi$ is a parameter characterizing the anti-FZZT brane, which is now taken to be common to all $K$ branes. The potential could have been normalized as

$$
\frac{1}{g_{\mathrm{s}}} V(H)
$$

where $g_{\mathrm{s}}$ is the genus counting parameter, so that the genus expansion is manifest. In this paper we include $g_{\mathrm{s}}^{-1}$ in $V$ for simplicity. In the double scaling limit, $N$ is sent to infinity and the potential turns into the effective potential. In this paper we will further take the 't Hooft limit

$$
K \rightarrow \infty, g_{\mathrm{s}} \rightarrow 0 \quad \text { with } t \equiv g_{\mathrm{s}} K \text { fixed }
$$

\footnotetext{
${ }^{3}$ In this paper we will eventually restrict ourselves to the JT gravity case, but most parts of our formalism can be applied to other $2 \mathrm{~d}$ gravities as well.
} 
and evaluate quantities in the planar approximation. That is, we will ignore all higher-order corrections of expansions in $g_{\mathrm{s}}$ and $K^{-1}$.

The matrices $H, Q$ are often denoted by their components $H_{a b}, Q_{a i}$, where $a, b=$ $1, \ldots, N$ are "color" indices and $i, j=1, \ldots, K$ are "flavor" indices. The color degrees of freedom are used for describing bulk gravity while the flavor degrees of freedom are thought of as describing the interior partners of the early Hawking radiation. One can regard the matrix element $H_{a b}$ as

$$
H_{a b}=\langle a|H| b\rangle,
$$

where $H$ is a Hamiltonian operator and $\{|a\rangle\}_{a=1}^{N}$ form an orthonormal basis of the corresponding $N$ dimensional Hilbert space

$$
\langle a \mid b\rangle=\delta_{a b}, \quad 1=\sum_{a}|a\rangle\langle a| .
$$

For $i$ th random vector variable $Q_{a i}$ we consider the (canonical) thermal pure quantum state $[31,32]$

$$
\left|\psi_{i}\right\rangle=\sum_{a} e^{-\frac{1}{2} \beta H}|a\rangle Q_{a i}=\sum_{a, b}|b\rangle\left(e^{-\frac{1}{2} \beta H}\right)_{b a} Q_{a i}
$$

Here $\beta$ is the inverse temperature, which is identified with the (renormalized) length of an asymptotic boundary in $2 \mathrm{~d}$ gravity. $\left|\psi_{i}\right\rangle$ play the role of the black hole microstates.

\section{$2.2 \quad$ Ensemble average}

To study the entropy, we will compute the average of overlaps such as $\left\langle\psi_{i} \mid \psi_{j}\right\rangle$. We define the average of $\mathcal{O}$ by

$$
\langle\overline{\mathcal{O}}\rangle=\int d H d Q d Q^{\dagger} e^{-\operatorname{Tr} V(H)-\operatorname{Tr} Q^{\dagger}(\xi+H) Q} \mathcal{O} .
$$

Here the angle brackets \langle\rangle represent averaging over the color degrees of freedom while the overline - represents averaging over the flavor degrees of freedom. It is convenient to change the variable as

$$
Q=(\xi+H)^{-\frac{1}{2}} C,
$$

so that the new random variable $C$ obeys the Gaussian distribution

$$
\langle\overline{\mathcal{O}}\rangle=\int d H d C d C^{\dagger} \operatorname{det}(\xi+H)^{-K} e^{-\operatorname{Tr} V(H)-\operatorname{Tr} C^{\dagger} C} \mathcal{O} .
$$

Thus, in terms of $C$ the flavor average becomes nothing but the Gaussian average. Note that the determinant factor is recovered from the integration measure. On the other hand, the thermal pure quantum state (2.6) becomes (see also appendix D of [13])

$$
\left|\psi_{i}\right\rangle=\sum_{a, b}|b\rangle\left[e^{-\frac{1}{2} \beta H}(\xi+H)^{-\frac{1}{2}}\right]_{b a} C_{a i} .
$$

For our discussion it is convenient to express (2.10) as

$$
\left|\psi_{i}\right\rangle=\sum_{a, b}|b\rangle(\sqrt{A})_{b a} C_{a i}
$$


with

$$
A(H)=\frac{e^{-\beta H}}{\xi+H}
$$

We then consider the overlaps such as

$$
\begin{aligned}
W_{i j} & \equiv\left\langle\psi_{i} \mid \psi_{j}\right\rangle=\sum_{a, b} A_{a b} C_{a i}^{*} C_{b j}, \\
W_{i j} W_{j i} & =\left|\left\langle\psi_{i} \mid \psi_{j}\right\rangle\right|^{2}=\sum_{a, b, a^{\prime}, b^{\prime}} A_{a b} A_{b^{\prime} a^{\prime}} C_{a i}^{*} C_{b j} C_{a^{\prime} i} C_{b^{\prime} j}^{*} .
\end{aligned}
$$

Recall that the Gaussian average of $C$ can be computed by the Wick contraction

$$
\begin{aligned}
\overline{C_{a i}^{*} C_{b j}} & =\delta_{a b} \delta_{i j}, \\
\overline{C_{a i}^{*} C_{b j} C_{a^{\prime} i} C_{b^{\prime} j}^{*}} & =\delta_{i j} \delta_{a b} \delta_{a^{\prime} b^{\prime}}+\delta_{a a^{\prime}} \delta_{b b^{\prime}} .
\end{aligned}
$$

By using these formulas, the average of the overlaps (2.13) are given by

$$
\begin{aligned}
\overline{\left\langle\psi_{i} \mid \psi_{j}\right\rangle} & =\delta_{i j} \operatorname{Tr} A, \\
\overline{\left|\left\langle\psi_{i} \mid \psi_{j}\right\rangle\right|^{2}} & =\delta_{i j}(\operatorname{Tr} A)^{2}+\operatorname{Tr} A^{2} .
\end{aligned}
$$

As discussed in [13], one can visualize the above computation (2.15) by drawing diagrams. For instance, $\left\langle\psi_{i} \mid \psi_{j}\right\rangle$ in (2.13) can be represented by the following diagram

$$
\left\langle\psi_{i} \mid \psi_{j}\right\rangle=\left(C^{\dagger} A C\right)_{i j}=\quad \stackrel{i}{i}\left(C^{\dagger}\right)_{i a} A_{a b} \quad C_{b j} j
$$

The black thick curve labeled by the color matrix $A_{a b}$ corresponds to the asymptotic boundary of $2 \mathrm{~d}$ spacetime while the dashed lines correspond to the flavor degrees of freedom $C, C^{\dagger}$. The gravitational path integral in the presence of branes is given by the matrix integral (2.9). One can easily see that the gravitational computations in eq. (2.10) and figure 3 of [13] agree with the first and the second lines of (2.15), respectively.

\subsection{Reduced density matrix of radiation}

As explained in [13], the reduced density matrix of radiation is represented by the ensemble average of

$$
\varrho_{i j}=\frac{W_{i j}}{\sum_{i=1}^{K} W_{i i}}=\left(\frac{W}{\operatorname{Tr} W}\right)_{i j} .
$$

This is normalized as $\operatorname{Tr} \varrho=1$. Let us first consider the "purity" $\overline{\operatorname{Tr} \varrho^{2}}$ as an example. In the planar approximation, we can take the average of the numerator and the denominator of $\operatorname{Tr} \varrho^{2}$ independently

$$
\begin{aligned}
\overline{\operatorname{Tr} \varrho^{2}} \approx \frac{\overline{\operatorname{Tr} W^{2}}}{\overline{\operatorname{Tr}^{2}}} & =\frac{K(\operatorname{Tr} A)^{2}+K^{2} \operatorname{Tr} A^{2}}{(K \operatorname{Tr} A)^{2}} \\
& =\frac{1}{K}+\frac{\operatorname{Tr} A^{2}}{(\operatorname{Tr} A)^{2}} .
\end{aligned}
$$


Similarly, the average of $\operatorname{Tr} \varrho^{n}$ is approximated as

$$
\overline{\operatorname{Tr} \varrho^{n}} \approx \frac{\overline{\operatorname{Tr} W^{n}}}{\left(\overline{\operatorname{Tr} W)^{n}}\right.}=\frac{\overline{\operatorname{Tr} W^{n}}}{(K \operatorname{Tr} A)^{n}} .
$$

$\overline{\operatorname{Tr} W^{n}}$ in the numerator can be computed by using the Wick contraction of $C$ and $C^{\dagger}$. In the planar approximation one obtains

$$
\begin{aligned}
\overline{\operatorname{Tr} W} & =K \operatorname{Tr} A, \\
\overline{\operatorname{Tr} W^{2}} & =K(\operatorname{Tr} A)^{2}+K^{2} \operatorname{Tr} A^{2}, \\
\overline{\operatorname{Tr} W^{3}} & =K(\operatorname{Tr} A)^{3}+3 K^{2} \operatorname{Tr} A^{2} \operatorname{Tr} A+K^{3} \operatorname{Tr} A^{3}, \\
\overline{\operatorname{Tr} W^{4}} & =K(\operatorname{Tr} A)^{4}+6 K^{2} \operatorname{Tr} A^{2}(\operatorname{Tr} A)^{2}+2 K^{3}\left(\operatorname{Tr} A^{2}\right)^{2}+4 K^{3} \operatorname{Tr} A^{3} \operatorname{Tr} A+K^{4} \operatorname{Tr} A^{4} .
\end{aligned}
$$

In fact, $\overline{\operatorname{Tr} W^{n}}$ can be computed efficiently by means of the generating function

$$
R(\lambda)=\operatorname{Tr} \frac{1}{\lambda-\varrho}=\sum_{n=0}^{\infty} \frac{\operatorname{Tr} \varrho^{n}}{\lambda^{n+1}}=\frac{K}{\lambda}+\sum_{n=1}^{\infty} \frac{\overline{\operatorname{Tr} W^{n}}}{\lambda^{n+1}(K \operatorname{Tr} A)^{n}}
$$

In the planar approximation $R(\lambda)$ satisfies

$$
\lambda R(\lambda)=K+\sum_{n=1}^{\infty} \frac{R(\lambda)^{n} \operatorname{Tr} A^{n}}{(K \operatorname{Tr} A)^{n}} .
$$

This equation was derived diagrammatically in [13]. We give an alternative derivation based on the saddle point method in appendix A. By plugging (2.21) into (2.22), $\overline{\operatorname{Tr} W^{n}}$ can be obtained recursively. In this way, in the planar approximation we obtain

$$
\left\langle\overline{\operatorname{Tr} \varrho^{n}}\right\rangle \approx \frac{1}{K^{n-1}}+\frac{n(n-1)}{2 K^{n-2}} \frac{\left\langle\operatorname{Tr} A^{2}\right\rangle}{\langle\operatorname{Tr} A\rangle^{2}}+\cdots+\frac{n}{K} \frac{\left\langle\operatorname{Tr} A^{n-1}\right\rangle}{\langle\operatorname{Tr} A\rangle^{n-1}}+\frac{\left\langle\operatorname{Tr} A^{n}\right\rangle}{\langle\operatorname{Tr} A\rangle^{n}} .
$$

Thus, to compute $\left\langle\overline{\operatorname{Tr} \varrho^{n}}\right\rangle$ we need to evaluate

$$
\left\langle\operatorname{Tr} A^{n}\right\rangle=\int d H e^{-\operatorname{Tr} V(H)} \operatorname{det}(\xi+H)^{-K} \operatorname{Tr} A^{n} .
$$

We evaluate it in the double scaling limit. In the planar approximation we have only to consider the genus zero part. It can be expressed in terms of the leading-order density $\rho_{0}(E)$ of the eigenvalues of $H$. As we studied in [24], for Witten-Kontsevich topological gravity with general couplings $\left\{t_{k}\right\}\left(k \in \mathbb{Z}_{\geq 0}\right), \rho_{0}(E)$ is given by

$$
\rho_{0}(E)=\frac{1}{\sqrt{2} \pi g_{\mathrm{s}}} \int_{E_{0}}^{E} \frac{d v}{\sqrt{E-v}} \frac{\partial f(-v)}{\partial(-v)}
$$

with

$$
f(u):=\sum_{k=0}^{\infty}\left(\delta_{k, 1}-t_{k}\right) \frac{u^{k}}{k !} .
$$


The threshold energy $E_{0}$ is determined by the condition (the genus-zero string equation)

$$
f\left(-E_{0}\right)=0
$$

In this paper we consider JT gravity, which corresponds to a particular background $t_{k}=\gamma_{k}$ with $[33-35]^{4}$

$$
\gamma_{0}=\gamma_{1}=0, \quad \gamma_{k}=\frac{(-1)^{k}}{(k-1) !} \quad(k \geq 2)
$$

As we studied in [24], the effect of anti-FZZT branes, i.e. the insertion of $\operatorname{det}(\xi+H)^{-K}$ amounts to shifting the couplings $t_{k}$ of topological gravity as ${ }^{5}$

$$
t_{k}=\gamma_{k}+t(2 k-1) ! !(2 \xi)^{-k-\frac{1}{2}} .
$$

This is valid as long as $\operatorname{Re} \xi>0$. Here $t$ is the 't Hooft coupling in (2.3). Thus, (2.24) is evaluated as

$$
\begin{aligned}
\left\langle\operatorname{Tr} A^{n}\right\rangle & \approx\left\langle\operatorname{Tr} A^{n}\right\rangle^{g=0} \\
& =\int_{E_{0}}^{\infty} d E \rho_{0}(E) A(E)^{n} \\
& \equiv Z_{n}
\end{aligned}
$$

where $\rho_{0}(E)$ is now evaluated in the background (2.29). In this background, (2.26) becomes

$$
f(u=-v) \equiv f(v, t)=\sqrt{v} I_{1}(2 \sqrt{v})+\frac{t}{\sqrt{2 \xi+2 v}},
$$

where we have changed the variable as $v=-u$ for convenience and $I_{k}(z)$ denotes the modified Bessel function of the first kind. (2.25) then becomes

$$
\rho_{0}(E)=\frac{1}{\sqrt{2} \pi g_{\mathrm{s}}}\left(\int_{E_{0}}^{E} d v \frac{I_{0}(2 \sqrt{v})}{\sqrt{E-v}}-\frac{t}{E+\xi} \sqrt{\frac{E-E_{0}}{2\left(E_{0}+\xi\right)}}\right) .
$$

Note that in [24] we calculated $\rho_{0}(E)$ for JT gravity in the presence of $K$ FZZT branes. The above $\rho_{0}(E)$ for anti-FZZT branes is essentially identical to this except the sign of the 't Hooft coupling $t$. Note also that this expression of $\rho_{0}(E)$ is valid as long as $t$ is not greater than the critical value $t_{\mathrm{c}}$. We will explain this in section 3.1.

We emphasize that we have treated anti-FZZT branes as dynamical objects. More specifically, in (2.24) the color average is evaluated in the presence of the determinant factor $\operatorname{det}(\xi+H)^{-K}$ and as a consequence the deformed eigenvalue density (2.32) is used

\footnotetext{
${ }^{4}$ Another way to obtain JT gravity is to take the $p \rightarrow \infty$ limit of the $(2, p)$ minimal string $[21,36]$. Entanglement entropy in this context was studied recently in [37].

${ }^{5}$ This shift of couplings was first recognized in the theory of soliton equations [38] and appears in various contexts of matrix models and related subjects. For more details, see [24] and references therein.
} 
in (2.30). This is the main difference from the approach of [13], which is based on the probe brane approximation at genus-zero

$$
\begin{aligned}
\left.\left\langle\operatorname{Tr} A^{n}\right\rangle_{\text {probe }}\right|_{g=0} & =\left.\int d H e^{-\operatorname{Tr} V(H)} \operatorname{Tr} A^{n}\right|_{g=0} \\
& =\int_{0}^{\infty} d E \rho_{0}^{\mathrm{JT}}(E) A(E)^{n},
\end{aligned}
$$

where the original JT gravity density of state $\rho_{0}^{\mathrm{JT}}(E)$ is given by

$$
\rho_{0}^{\mathrm{JT}}(E)=\frac{\sinh (2 \sqrt{E})}{\sqrt{2} \pi g_{\mathrm{s}}} .
$$

However, in [13] the same 't Hooft limit as ours (2.3) is used. As we argued in [24], in this limit the back-reaction of (anti-)FZZT branes cannot be ignored and the couplings $t_{k}$ are shifted due to the insertion of branes. As a consequence, the eigenvalue density is deformed from $\rho_{0}^{\mathrm{JT}}(E)$ in $(2.34)$ to $\rho_{0}(E)$ in $(2.32)$. Thus we have to use the dynamical brane picture in this limit.

\subsection{Resolvent of reduced density matrix and entropy}

We saw in the last subsection that the ensemble averages of $\varrho$ in the planar approximation are expressed in terms of $Z_{n}$ in (2.30). On the other hand, the general expression (2.23) of $\left\langle\overline{\operatorname{Tr} \varrho^{n}}\right\rangle$ is rather complicated as a function of $n$ and it is difficult to apply the replica trick directly to (2.23) to calculate the entropy. Instead, as detailed in [13], we can study the entropy using the resolvent $R(\lambda)$ for $\varrho$ in (2.21). By substituting (2.30), the SchwingerDyson equation (2.22) for $R(\lambda)$ becomes

$$
\begin{aligned}
\lambda R(\lambda) & =K+\sum_{n=1}^{\infty} \frac{R(\lambda)^{n}}{\left(K Z_{1}\right)^{n}} \int_{E_{0}}^{\infty} d E \rho_{0}(E) A(E)^{n} \\
& =K+\int_{E_{0}}^{\infty} d E \rho_{0}(E) \frac{w(E) R(\lambda)}{K-w(E) R(\lambda)}
\end{aligned}
$$

where we have defined

$$
w(E)=\frac{A(E)}{Z_{1}} .
$$

Following [13], we divide the integral in (2.35) into two pieces

$$
\begin{aligned}
\lambda R(\lambda) & =K+\int_{E_{0}}^{E_{K}} d E \rho_{0}(E) \frac{w(E) R(\lambda)}{K-w(E) R(\lambda)}+\int_{E_{K}}^{\infty} d E \rho_{0}(E) \frac{w(E) R(\lambda)}{K-w(E) R(\lambda)} \\
& \approx K+\int_{E_{0}}^{E_{K}} d E \rho_{0}(E) \frac{w(E) R(\lambda)}{K-w(E) R(\lambda)}+\lambda_{0} R(\lambda)
\end{aligned}
$$

where $\lambda_{0}$ and $E_{K}$ are defined by

$$
\begin{aligned}
\lambda_{0} & =\frac{1}{K} \int_{E_{K}}^{\infty} d E \rho_{0}(E) w(E), \\
K & =\int_{E_{0}}^{E_{K}} d E \rho_{0}(E) .
\end{aligned}
$$


By rewriting (2.37) as

$$
R(\lambda)=\frac{K}{\lambda-\lambda_{0}}+\frac{1}{\lambda-\lambda_{0}} \int_{E_{0}}^{E_{K}} d E \rho_{0}(E) \frac{w(E) R(\lambda)}{K-w(E) R(\lambda)},
$$

we can solve $R(\lambda)$ by the iteration starting from $R(\lambda)=K /\left(\lambda-\lambda_{0}\right)$. As discussed in [13], the second order iteration gives

$$
\begin{aligned}
R(\lambda) & \approx \frac{K}{\lambda-\lambda_{0}}+\frac{1}{\lambda-\lambda_{0}} \int_{E_{0}}^{E_{K}} d E \rho_{0}(E) \frac{w(E) K\left(\lambda-\lambda_{0}\right)^{-1}}{K-w(E) K\left(\lambda-\lambda_{0}\right)^{-1}} \\
& =\frac{K}{\lambda-\lambda_{0}}+\frac{1}{\lambda-\lambda_{0}} \int_{E_{0}}^{E_{K}} d E \rho_{0}(E) \frac{w(E)}{\lambda-\lambda_{0}-w(E)} .
\end{aligned}
$$

Using (2.38), we find

$$
\begin{aligned}
R(\lambda) & =\frac{1}{\lambda-\lambda_{0}}\left[\int_{E_{0}}^{E_{K}} d E \rho_{0}(E)+\int_{E_{0}}^{E_{K}} d E \rho_{0}(E) \frac{w(E)}{\lambda-\lambda_{0}-w(E)}\right] \\
& =\int_{E_{0}}^{E_{K}} d E \rho_{0}(E) \frac{1}{\lambda-\lambda_{0}-w(E)} .
\end{aligned}
$$

The eigenvalue density $D(\lambda)$ of the density matrix $\varrho_{i j}$ is obtained from the discontinuity of $R(\lambda)$

$$
D(\lambda)=\frac{R(\lambda-\mathrm{i} 0)-R(\lambda+\mathrm{i} 0)}{2 \pi \mathrm{i}}=\int_{E_{0}}^{E_{K}} d E \rho_{0}(E) \delta\left(\lambda-\lambda_{0}-w(E)\right) .
$$

Finally, the von Neumann entropy is given by

$$
\begin{aligned}
S & =-\int d \lambda D(\lambda) \lambda \log \lambda \\
& =-\int_{E_{0}}^{E_{K}} d E \rho_{0}(E)\left(\lambda_{0}+w(E)\right) \log \left(\lambda_{0}+w(E)\right) .
\end{aligned}
$$

We will use this expression to study the Page curve numerically in section 4 .

In the rest of this section let us make several comments on the above approximation. We can check that $D(\lambda)$ in $(2.42)$ is normalized correctly

$$
\operatorname{Tr} \varrho^{0}=\int d \lambda D(\lambda) \cdot 1=\int_{E_{0}}^{E_{K}} d E \rho_{0}(E)=K,
$$

where we have used (2.38). We also find

$$
\begin{aligned}
\operatorname{Tr} \varrho & =\int d \lambda D(\lambda) \cdot \lambda=\int_{E_{0}}^{E_{K}} d E \rho_{0}(E)\left(\lambda_{0}+w(E)\right) \\
& =K \lambda_{0}+\int_{E_{0}}^{E_{K}} d E \rho_{0}(E) w(E) \\
& =\int_{E_{K}}^{\infty} d E \rho_{0}(E) w(E)+\int_{E_{0}}^{E_{K}} d E \rho_{0}(E) w(E) \\
& =\int_{E_{0}}^{\infty} d E \rho_{0}(E) w(E) \\
& =\frac{1}{Z_{1}} \int_{E_{0}}^{\infty} d E \rho_{0}(E) A(E)=\frac{1}{Z_{1}} Z_{1}=1,
\end{aligned}
$$

where we have used (2.38). 
$\lambda_{0}$ in $(2.38)$ can be written as

$$
\begin{aligned}
\lambda_{0} & =\frac{1}{K} \int_{E_{0}}^{\infty} d E \rho_{0}(E) w(E)-\frac{1}{K} \int_{E_{0}}^{E_{K}} d E \rho_{0}(E) w(E) \\
& =\frac{1}{K}-\bar{w}_{K},
\end{aligned}
$$

where we have used (2.45) and defined

$$
\bar{w}_{K} \equiv \frac{1}{K} \int_{E_{0}}^{E_{K}} d E \rho_{0}(E) w(E)=\frac{\int_{E_{0}}^{E_{K}} d E \rho_{0}(E) w(E)}{\int_{E_{0}}^{E_{K}} d E \rho_{0}(E)} .
$$

That is, $\bar{w}_{K}$ is the average of $w(E)$ in the "post Page" subspace $E<E_{K}$. Thus the resolvent is written as

$$
R(\lambda)=\operatorname{Tr} \frac{1}{\lambda-\varrho}=\int_{E_{0}}^{E_{K}} d E \rho_{0}(E) \frac{1}{\lambda-\lambda(E)},
$$

where

$$
\lambda(E)=\frac{1}{K}+w(E)-\bar{w}_{K} .
$$

$\lambda(E)$ behaves as

$$
\lambda(E) \approx \begin{cases}\frac{1}{K} & \left(K \ll g_{\mathrm{s}}^{-1}\right), \\ w(E)-\bar{w}_{K} & \left(K \gg g_{\mathrm{s}}^{-1}\right) .\end{cases}
$$

This corresponds to figure 6 in [13]. Note that the density matrix $\varrho_{i j}$ is originally a matrix in the flavor space, but after taking the average the spectrum $\lambda(E)$ of $\varrho_{i j}$ is effectively written in terms of energy eigenvalues in the "color" space. We have to project quantities onto the "post Page" subspace $E<E_{K}$ to ensure that the number of total state is $K=$ $\int_{E<E_{K}} d E \rho_{0}(E)$.

\section{Phase transition}

An interesting feature of the anti-FZZT brane background in JT gravity is that the system exhibits a phase transition as the 't Hooft coupling $t$ varies. In this section we discuss this phase transition and study the critical behavior of the entropy.

\subsection{Threshold energy}

Let us first clarify the definition of the threshold energy $E_{0}$. In the last section we saw that $E_{0}$ is determined by the threshold energy condition (2.27). For JT gravity with anti-FZZT branes, $f$ is given by (2.31) and the condition (2.27) is written explicitly as

$$
\sqrt{E_{0}} I_{1}\left(2 \sqrt{E_{0}}\right)=-\frac{t}{\sqrt{2\left(E_{0}+\xi\right)}} .
$$

The threshold energy $E_{0}$ is determined as a real solution of this equation. Here, $t>0$ by definition and we take $\xi>0$ in order for the shift of the couplings (2.29) to be valid. We 


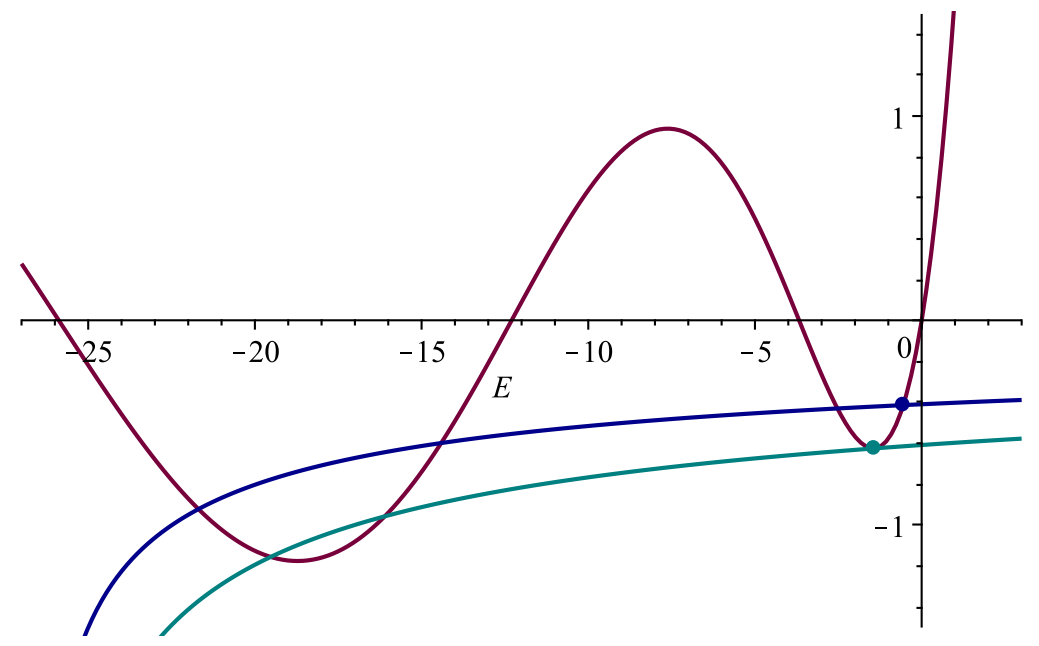

Figure 1. The threshold energy $E_{0}$ is determined by the equation (3.1). The red curve represents the graph of $\sqrt{E} I_{1}(2 \sqrt{E})$ while the blue and green curves are the graphs of $-t / \sqrt{2(E+\xi)}$ with $t=3$ and $t=t_{\mathrm{c}} \approx 4.46$ respectively, where we set $\xi=27$. As we see in this example, (3.1) could have multiple real solutions. The threshold energy $E_{0}$ is determined as the largest real solution, as indicated by a dot. The horizontal location of the green dot gives the critical value $E_{0}^{c}$.

show the plots of both sides of the equation (3.1) in figure 1. We see that $\sqrt{E} I_{1}(2 \sqrt{E})$ oscillates for $E<0$ and increases monotonically for $E>0$ starting from the origin, while $-t / \sqrt{2(E+\xi)}$ is always negative. Therefore $E_{0}$ has to be negative if it exists. However, the number of real solutions of (3.1) varies depending on the values of $t$ and $\xi$. In particular, (3.1) has no real solution when $t$ is very large, whereas it has multiple real solutions when $\xi$ is large and $t$ is small. ${ }^{6}$ On the other hand, one can easily see that (3.1) always has at least one real solution for sufficiently small $t$. We define $E_{0}$ as the largest real solution of (3.1) (i.e. the solution with the smallest absolute value), so that it is continuously deformed from $E_{0}=0$ for the original JT gravity case $t=0$.

As we see from figure $1,\left|E_{0}\right|$ is small for small $t$. If we increase $t,\left|E_{0}\right|$ also increases. Then there exists a critical point $t=t_{\mathrm{c}}$ beyond which $E_{0}$ no longer continues as a real solution. Thus, we expect a phase transition. This transition is qualitatively very similar to the one discussed in [25] in the case of EOW branes. If one continuously increases $t$ beyond the critical point, $E_{0}$ and the second largest root turn into a pair of complex roots. It is therefore very likely that the saddle point of the matrix integral is described by an eigenvalue density with "Y" shaped support, similar to the one studied in [25]. It would be interesting to study the model in this "Y" shaped phase further. In this paper we view this phase transition as the end of the black hole evaporation and focus on the physics before the phase transition.

\footnotetext{
${ }^{6}$ See also [39] for a similar problem in JT gravity with conical defects.
} 


\subsection{Behavior of threshold energy near $t=t_{\mathrm{c}}$}

At the critical value $t=t_{\mathrm{c}}$, the equation (3.1) has a double root $E_{0}^{\mathrm{c}}$. Thus $t_{\mathrm{c}}$ is determined by the condition

$$
f\left(E_{0}^{\mathrm{c}}, t_{\mathrm{c}}\right)=0, \quad \partial_{v} f\left(E_{0}^{\mathrm{c}}, t_{\mathrm{c}}\right)=0
$$

with $f(v, t)$ given in (2.31). Expanding the equation $f\left(E_{0}, t\right)=0$ around $(v, t)=\left(E_{0}^{\mathrm{c}}, t_{\mathrm{c}}\right)$, we find

$$
\begin{aligned}
0= & f\left(E_{0}^{\mathrm{c}}, t_{\mathrm{c}}\right)+\partial_{v} f\left(E_{0}^{\mathrm{c}}, t_{\mathrm{c}}\right)\left(E_{0}-E_{0}^{\mathrm{c}}\right)+\frac{1}{2} \partial_{v}^{2} f\left(E_{0}^{\mathrm{c}}, t_{\mathrm{c}}\right)\left(E_{0}-E_{0}^{\mathrm{c}}\right)^{2} \\
& +\partial_{t} f\left(E_{0}^{\mathrm{c}}, t_{\mathrm{c}}\right)\left(t-t_{\mathrm{c}}\right)+\cdots .
\end{aligned}
$$

The first two terms vanish due to (3.2). Thus, near $t=t_{\mathrm{c}}$ we find

$$
E_{0}-E_{0}^{\mathrm{c}} \approx C \sqrt{t_{\mathrm{c}}-t} \quad\left(t<t_{\mathrm{c}}\right),
$$

where $C$ is given by

$$
C=\sqrt{\frac{2 \partial_{t} f\left(E_{0}^{\mathrm{c}}, t_{\mathrm{c}}\right)}{\partial_{v}^{2} f\left(E_{0}^{\mathrm{c}}, t_{\mathrm{c}}\right)}}
$$

\subsection{Effective zero-temperature entropy and von Neumann entropy near $t=t_{\mathrm{c}}$}

In [25], the effective zero-temperature entropy $S_{\text {eff }}$ was introduced. It is defined by the behavior of $\rho_{0}(E)$ near $E=E_{0}$ :

$$
\rho_{0}(E) \sim e^{S_{\text {eff }}} \sqrt{E-E_{0}}
$$

From $(2.25)$ we find

$$
\begin{aligned}
e^{S_{\mathrm{eff}}} & =\frac{\sqrt{2}}{\pi g_{\mathrm{s}}} \partial_{v} f\left(E_{0}, t\right) \\
& =\frac{\sqrt{2}}{\pi g_{\mathrm{s}}}\left[I_{0}\left(2 \sqrt{E_{0}}\right)-\frac{t}{\left(2 \xi+2 E_{0}\right)^{\frac{3}{2}}}\right] .
\end{aligned}
$$

In figure 2 , we show the plot of $S_{\text {eff. }}$. We see that $S_{\text {eff }}$ is a monotonically decreasing function of $t$. Near $t=t_{\mathrm{c}}$, using (3.2) and (3.4) we find

$$
\begin{aligned}
e^{S_{\mathrm{eff}}} & \sim \partial_{v} f\left(E_{0}, t\right) \\
& =\partial_{v} f\left(E_{0}^{\mathrm{c}}, t_{\mathrm{c}}\right)+\partial_{v}^{2} f\left(E_{0}^{\mathrm{c}}, t_{\mathrm{c}}\right)\left(E_{0}-E_{0}^{\mathrm{c}}\right)+\partial_{t} \partial_{v} f\left(E_{0}^{\mathrm{c}}, t_{\mathrm{c}}\right)\left(t-t_{\mathrm{c}}\right)+\cdots \\
& \sim \sqrt{t_{\mathrm{c}}-t} .
\end{aligned}
$$

Using the above results, we can evaluate the critical behavior of the von Neumann entropy (2.43). It turns out that the critical behavior of the von Neumann entropy $S(t)$ in (2.43) is determined by that of the eigenvalue density near $E=E_{0}$

$$
\rho_{0}(E) \sim e^{S_{\text {eff }}} \sqrt{E-E_{0}} \sim \sqrt{t_{\mathrm{c}}-t} \sqrt{E-E_{0}} .
$$

One can show that the contribution of the $E$-integral (2.43) away from the edge $E=E_{0}$ is finite at $t=t_{\mathrm{c}}$. Subtracting this finite contribution and using (3.9) near $E=E_{0}$ in (2.43), we find

$$
S(t)-S\left(t_{\mathrm{c}}\right) \sim \sqrt{t_{\mathrm{c}}-t} .
$$

In the next section we will confirm this behavior numerically. 


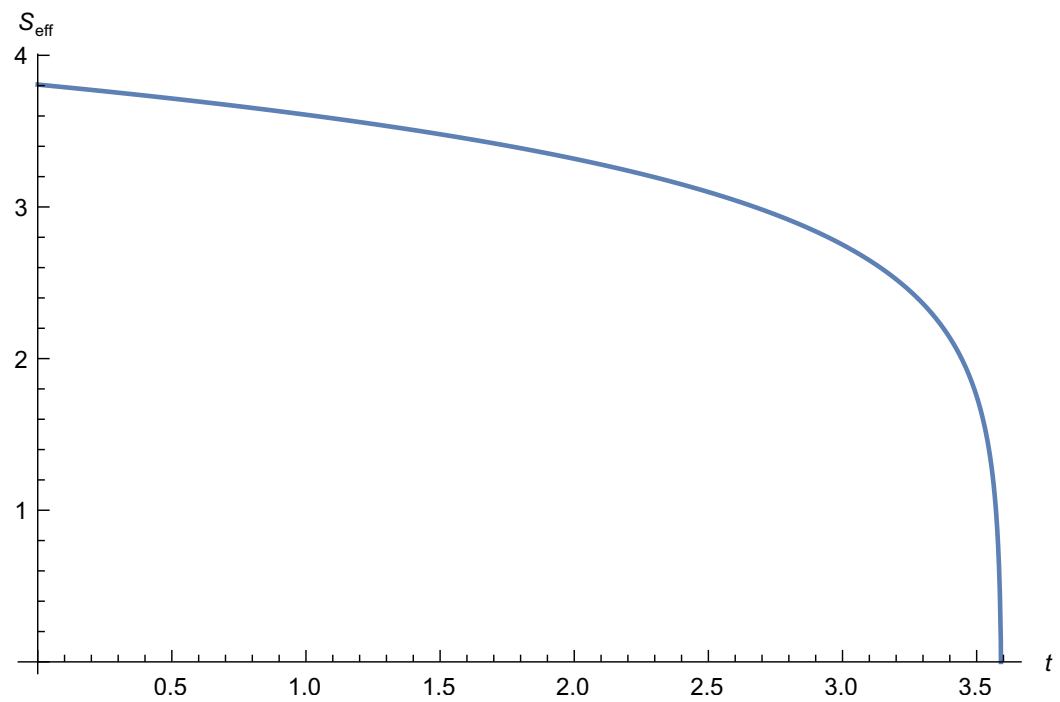

Figure 2. Plot of the effective entropy $S_{\text {eff. }}$ We set $\xi=18, g_{\mathrm{s}}=1 / 100$ in this figure.

\section{Numerical study of Page curve}

In section 2 we saw how to calculate the entropy. In this section we will numerically study the Page curve, i.e. the time evolution of the entropy. In Page's original calculation $\log K$ is regarded as "time" [19]. Since we take the 't Hooft limit (2.3), we will regard log $t$ as "time". We will plot the entropy as a function of $t$ rather than $\log t$, which is convenient for seeing the critical behavior discussed in section 3 .

\section{1 von Neumann entropy}

We consider the von Neumann entropy (2.43) in JT gravity in the presence of $K$ antiFZZT branes. As discussed in section 2 we compute the entropy for dynamical branes, but it is interesting to compute it in the probe brane approximation as well for the sake of comparison. In the probe brane approximation, we have $E_{0}=0$ and the eigenvalue density is given by $\rho_{0}^{\mathrm{JT}}(E)$ in $(2.34)$. We show the plot of the von Neumann entropy $S$ in figure 3. We see that the entropy for the dynamical brane (solid blue curve) starts to decrease relative to the probe brane case (dashed orange curve). This is very similar to the Page curve of an evaporating black hole. As we saw in the last section, we observe that the entropy exhibits the critical behavior (3.10).

\subsection{Rényi entropy}

It is also interesting to consider the Rényi entropy. The $n$th Rényi entropy $S_{n}$ is defined by

$$
\left\langle\overline{\operatorname{Tr} \varrho^{n}}\right\rangle=e^{-(n-1) S_{n}} .
$$

For large $K,\left\langle\overline{\operatorname{Tr} \varrho^{n}}\right\rangle$ is dominated by the last term of (2.23). Thus as $t$ grows, $S_{n}$ approaches

$$
\widetilde{S}_{n}:=-\frac{1}{n-1} \log \frac{Z_{n}}{\left(Z_{1}\right)^{n}} .
$$




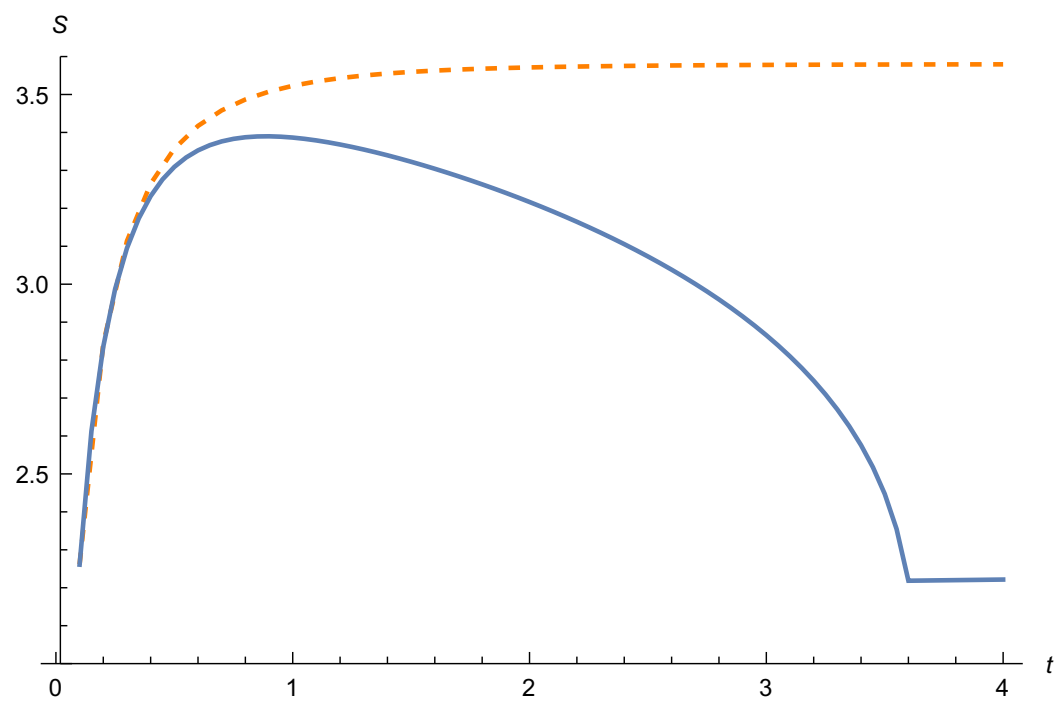

Figure 3. Plot of the von Neumann entropy $S$ in (2.43) as a function of $t=g_{\mathrm{s}} K$. We set $\xi=18, \beta=4, g_{\mathrm{s}}=1 / 100$ in this figure. The solid blue curve is the dynamical brane case while the dashed orange curve is the probe brane case.

Let us first consider the second Rényi entropy

$$
S_{2}=-\log \left(\frac{g_{\mathrm{s}}}{t}+\frac{Z_{2}}{Z_{1}^{2}}\right)
$$

In figure 4 , we show the plot of $S_{2}$ as a function of $t$. We can see that $S_{2}$ first increases and then decreases. Near $t=t_{\mathrm{c}}, S_{2}$ exhibits a critical behavior

$$
S_{2}(t)-S_{2}\left(t_{\mathrm{c}}\right) \sim \sqrt{t_{\mathrm{c}}-t} .
$$

This behavior can be derived in the same way as in the case of the von Neumann entropy.

We can see that the plot of $S_{2}$ has a similar behavior with the Page curve of Hawking radiation from an evaporating black hole (see e.g. figure 7 in [15]). We regard $\widetilde{S}_{2}$ as an analogue of the thermodynamic entropy $S_{\mathrm{BH}}$ of an evaporating black hole. From figure 4 , we see that $\widetilde{S}_{2}$ is a monotonically decreasing function of $t$ (represented by the dashed orange curve).

Let us next consider the third Rényi entropy

$$
e^{-2 S_{3}}=\frac{1}{K^{2}}+\frac{3 Z_{2}}{K Z_{1}^{2}}+\frac{Z_{3}}{Z_{1}^{3}}
$$

As $t$ grows, this approaches

$$
e^{-2 \widetilde{S}_{3}}=\frac{Z_{3}}{Z_{1}^{3}}
$$

In figure 5 we show the plot of $S_{3}$ for anti-FZZT branes. We see that this is qualitatively very similar to the $S_{2}$ case. In particular, $S_{3}$ is bounded from above by $\widetilde{S}_{3}$, which monotonically decreases. From figure 4 and 5, it is natural to regard $\widetilde{S}_{n}$ in (4.2) as an analogue of 




Figure 4. Plot of the second Rényi entropy $S_{2}$ in (4.3) as a function of $t=g_{\mathrm{s}} K$. We set $\xi=18, \beta=$ $4, g_{\mathrm{s}}=1 / 100$ in this figure. The solid blue curve is $S_{2}$ in (4.3). The dashed orange curve represents $\widetilde{S}_{2}$ in (4.2) while the green dashed curve is $S_{2}$ without taking account of the back-reaction.



Figure 5. Plot of the third Rényi entropy $S_{3}$ in (4.5) as a function of $t=g_{\mathrm{s}} K$. We set $\xi=18, \beta=$ $4, g_{\mathrm{s}}=1 / 100$ in this figure. The solid blue curve is $S_{3}$ in (4.5). The dashed orange curve represents $\widetilde{S}_{3}$ in $(4.6)$. 
the thermodynamic entropy $S_{\mathrm{BH}}$ of black hole, since $S_{\mathrm{BH}}$ decreases monotonically during the evaporation process as well

$$
\widetilde{S}_{n} \leftrightarrow S_{\mathrm{BH}}
$$

Based on the above numerical results, we conjecture that $\widetilde{S}_{n}$ defined in (4.2) is a monotonically decreasing function of $t$. More specifically, we conjecture that

$$
\partial_{t} \widetilde{S}_{n}<0 \quad \text { for } \quad n>1, \quad 0<t<t_{\mathrm{c}} .
$$

We will study this monotonic behavior in the next section.

\section{Monotonicity of $\widetilde{\boldsymbol{S}}_{n}$}

In this section we study the monotonically decreasing behavior of $\widetilde{S}_{n}$. We will prove our conjecture (4.8) in the large $\xi$ limit. We will also discuss how to understand intuitively this monotonically decreasing behavior.

\subsection{Leading-order eigenvalue density and its derivative}

In this subsection let us derive some useful formulas about the leading-order eigenvalue density $\rho_{0}(E)$ in $(2.25)$ for Witten-Kontsevich gravity with general couplings $\left\{t_{k}\right\}$, which we will use in the next subsection.

To study general Witten-Kontsevich gravity it is convenient to introduce the ItzyksonZuber variables [40]

$$
\mathcal{I}_{n}(u) \equiv \mathcal{I}_{n}\left(u,\left\{t_{k}\right\}\right)=\sum_{m=0}^{\infty} t_{n+m} \frac{u^{m}}{m !} \quad(n \geq 0)
$$

In terms of $\mathcal{I}_{n}, f(u)$ in $(2.26)$ is written as

$$
f(u)=u-\mathcal{I}_{0}(u)
$$

and the leading-order density $\rho_{0}(E)$ in $(2.25)$ becomes

$$
\rho_{0}(E)=\frac{1}{\sqrt{2} \pi g_{\mathrm{s}}} \int_{E_{0}}^{E} d v \frac{1-\mathcal{I}_{1}(-v)}{\sqrt{E-v}} .
$$

Let us now consider the anti-FZZT brane background (2.29). We are interested in how the entropy evolves as the 't Hooft coupling $t$ in (2.3) grows. Note that $t$ is implicitly related to $E_{0}$ by the string equation (2.27), from which one finds

$$
\begin{aligned}
0 & =\partial_{t} f\left(-E_{0}\right) \\
& =\left(\partial_{t} E_{0}\right)\left(\mathcal{I}_{1}\left(-E_{0}\right)-1\right)-\left.\partial_{t} \mathcal{I}_{0}(u)\right|_{u=-E_{0}} \\
& =\left(\partial_{t} E_{0}\right)\left(\mathcal{I}_{1}\left(-E_{0}\right)-1\right)-\frac{1}{\sqrt{2 \xi+2 E_{0}}} .
\end{aligned}
$$


By using this relation, the $t$-derivative of $\rho_{0}(E)$ is calculated as

$$
\begin{aligned}
\partial_{t} \rho_{0}(E) & =\left(\partial_{t} E_{0}\right) \partial_{E_{0}} \rho_{0}(E)-\frac{1}{\sqrt{2} \pi g_{\mathrm{s}}} \int_{E_{0}}^{E} d v \frac{\partial_{t} \mathcal{I}_{1}(-v)}{\sqrt{E-v}} \\
& =\frac{\partial_{t} E_{0}}{\sqrt{2} \pi g_{\mathrm{s}}} \frac{\mathcal{I}_{1}\left(-E_{0}\right)-1}{\sqrt{E-E_{0}}}-\frac{1}{\sqrt{2} \pi g_{\mathrm{s}}} \int_{E_{0}}^{E} d v(E-v)^{-\frac{1}{2}}(2 \xi+2 v)^{-\frac{3}{2}} \\
& =\frac{1}{2 \pi g_{\mathrm{s}} \sqrt{\left(E-E_{0}\right)\left(\xi+E_{0}\right)}}-\frac{1}{2 \pi g_{\mathrm{s}}(E+\xi)} \sqrt{\frac{E-E_{0}}{\xi+E_{0}}} \\
& =\frac{1}{2 \pi g_{\mathrm{s}}(E+\xi)} \sqrt{\frac{\xi+E_{0}}{E-E_{0}}} .
\end{aligned}
$$

Note that the background (2.29) is written for JT gravity, but we have never used the specific values of $\gamma_{k}$ in the above derivation. Therefore, (5.5) is in fact valid for the antiFZZT brane background of other gravities as well.

\subsection{Proof in the large $\xi$ limit}

In the last section we conjectured that $\widetilde{S}_{n}$ defined in (4.2) is a monotonically decreasing function of $t$. In this subsection let us prove this conjecture (4.8) at large $\xi$. From the expression (4.2), we see that (4.8) is equivalent to

$$
\frac{\partial_{t} Z_{n}}{n Z_{n}}>\frac{\partial_{t} Z_{1}}{Z_{1}}
$$

By using the property $\rho_{0}\left(E_{0}\right)=0$ and the expression (5.5), the $t$-derivative of $Z_{n}$ in (2.30) is calculated as

$$
\begin{aligned}
\partial_{t} Z_{n} & =\int_{E_{0}}^{\infty} d E \partial_{t} \rho_{0}(E) A(E)^{n} \\
& =\int_{E_{0}}^{\infty} d E \frac{1}{2 \pi g_{\mathrm{s}}} \sqrt{\frac{\xi+E_{0}}{E-E_{0}}} \frac{e^{-n \beta E}}{(E+\xi)^{n+1}} \\
& =\frac{\sqrt{\xi+E_{0}}}{2 \pi g_{\mathrm{s}}} e^{-n \beta E_{0}} \int_{0}^{\infty} d \widetilde{E} \widetilde{E}^{-\frac{1}{2}}\left(\widetilde{E}+E_{0}+\xi\right)^{-n-1} e^{-n \beta \widetilde{E}},
\end{aligned}
$$

where we have set $\widetilde{E}=E-E_{0}$. For large $\xi$, (5.7) is evaluated as

$$
\partial_{t} Z_{n}=\frac{e^{-n \beta E_{0}}}{2 \sqrt{n \pi \beta} g_{\mathrm{s}}} \xi^{-n-\frac{1}{2}}+\mathcal{O}\left(\xi^{-n-\frac{3}{2}}\right) .
$$

On the other hand, by plugging (2.32) into (2.30), $Z_{n}$ is written as

$$
\begin{aligned}
Z_{n}= & \int_{E_{0}}^{\infty} \rho_{0}(E) A(E)^{n} \\
= & \frac{1}{\sqrt{2} \pi g_{\mathrm{s}}} \int_{E_{0}}^{\infty} d v I_{0}(2 \sqrt{v}) \int_{v}^{\infty} d E(E-v)^{-\frac{1}{2}}(E+\xi)^{-n} e^{-n \beta E} \\
& -\frac{t}{2 \pi g_{\mathrm{s}}\left(E_{0}+\xi\right)^{\frac{1}{2}}} \int_{E_{0}}^{\infty} d E\left(E-E_{0}\right)^{\frac{1}{2}}(E+\xi)^{-n-1} e^{-n \beta E}
\end{aligned}
$$


In the same way as in (5.7)-(5.8), the above integrals at large $\xi$ are evaluated as

$$
\begin{aligned}
Z_{n}= & \frac{1}{\sqrt{2 n \pi \beta} g_{\mathrm{s}}} \int_{E_{0}}^{\infty} d v I_{0}(2 \sqrt{v})(v+\xi)^{-n} e^{-n \beta v}+\mathcal{O}\left(\xi^{-n-1}\right) \\
& -\frac{t e^{-n \beta E_{0}}}{4 \sqrt{\pi} g_{\mathrm{s}}(n \beta)^{\frac{3}{2}}} \xi^{-n-\frac{3}{2}}+\mathcal{O}\left(\xi^{-n-\frac{5}{2}}\right) \\
= & \frac{1}{\sqrt{2 n \pi \beta} g_{\mathrm{s}} \xi^{n}} \int_{E_{0}}^{\infty} d v I_{0}(2 \sqrt{v}) e^{-n \beta v}+\mathcal{O}\left(\xi^{-n-1}\right) .
\end{aligned}
$$

Here we see that in the leading-order of the large- $\xi$ approximation the first integral in (5.9) is dominant and the second integral does not contribute. Indeed, from (5.10) one can reproduce (5.8) by using the relation $\partial_{t} E_{0}=-\left(\sqrt{2 \xi} I_{0}\left(2 \sqrt{E_{0}}\right)\right)^{-1}+\mathcal{O}\left(\xi^{-3 / 2}\right)$, which follows from (3.1) or (5.4). Thus we obtain

$$
\frac{\partial_{t} Z_{n}}{n Z_{n}}=\frac{e^{-n \beta E_{0}}}{n \sqrt{2 \xi} \int_{E_{0}}^{\infty} d v I_{0}(2 \sqrt{v}) e^{-n \beta v}}+\mathcal{O}\left(\xi^{-\frac{3}{2}}\right) .
$$

To prove (5.6) at large $\xi$, it is sufficient to show that (5.11) monotonically increases as $n$ grows:

$$
\partial_{n} \frac{\partial_{t} Z_{n}}{n Z_{n}}>0
$$

Since $\partial_{t} Z_{n} / n Z_{n}>0,{ }^{7}$ this is equivalent to showing that

$$
\partial_{n} \log \frac{\partial_{t} Z_{n}}{n Z_{n}}>0
$$

The l.h.s. of (5.13) is rewritten as

$$
\begin{aligned}
\partial_{n} \log \frac{\partial_{t} Z_{n}}{n Z_{n}} & =-\beta E_{0}-\frac{1}{n}+\frac{\int_{E_{0}}^{\infty} d v I_{0}(2 \sqrt{v}) \beta v e^{-n \beta v}}{\int_{E_{0}}^{\infty} d v I_{0}(2 \sqrt{v}) e^{-n \beta v}} \\
& =\frac{\int_{E_{0}}^{\infty} d v I_{0}(2 \sqrt{v})\left[n \beta\left(v-E_{0}\right)-1\right] e^{-n \beta v}}{n \int_{E_{0}}^{\infty} d v I_{0}(2 \sqrt{v}) e^{-n \beta v}} \\
& =\frac{\int_{0}^{\infty} d \tilde{v} I_{0}\left(2 \sqrt{\tilde{v}+E_{0}}\right)(n \beta \tilde{v}-1) e^{-n \beta \tilde{v}}}{n e^{n \beta E_{0}} \int_{E_{0}}^{\infty} d v I_{0}(2 \sqrt{v}) e^{-n \beta v}},
\end{aligned}
$$

where we have set $\tilde{v}=v-E_{0}$. The denominator of the last expression in (5.14) is positive (see footnote 7). By renaming $\tilde{v}$ as $v$, the numerator is evaluated as

$$
\begin{aligned}
\int_{0}^{\infty} d v I_{0}\left(2 \sqrt{v+E_{0}}\right) n \beta v e^{-n \beta v}-\int_{0}^{\infty} d v I_{0}\left(2 \sqrt{v+E_{0}}\right) e^{-n \beta v} \\
=-\left.I_{0}\left(2 \sqrt{v+E_{0}}\right) v e^{-n \beta v}\right|_{0} ^{\infty}+\int_{0}^{\infty} d v\left(I_{0}\left(2 \sqrt{v+E_{0}}\right)+\frac{I_{1}\left(2 \sqrt{v+E_{0}}\right)}{\sqrt{v+E_{0}}} v\right) e^{-n \beta v} \\
\quad-\int_{0}^{\infty} d v I_{0}\left(2 \sqrt{v+E_{0}}\right) e^{-n \beta v} \\
=\int_{0}^{\infty} d v \frac{I_{1}\left(2 \sqrt{v+E_{0}}\right)}{\sqrt{v+E_{0}}} v e^{-n \beta v} .
\end{aligned}
$$

\footnotetext{
${ }^{7}$ This follows from (5.11) with $I_{0}(2 \sqrt{v})=\partial_{v}\left[\sqrt{v} I_{1}(2 \sqrt{v})\right]>0$ for $v>E_{0}^{\mathrm{c}}$, as we can see from figure 1.
} 
Since the integrand is positive for any $E_{0}$ satisfying $E_{0}^{\mathrm{c}}<E_{0}<0,{ }^{8}(5.15)$ is positive. Thus we have proved (5.13). Hence (4.8) has been proved at large $\xi$.

\subsection{Intuitive understanding of the decreasing behavior of $\widetilde{S}_{n}$}

Beyond the large $\xi$ approximation, it does not seem easy to find a simple analytic proof of the monotonically decreasing behavior of $\widetilde{S}_{n}$. Alternatively, in this subsection we will explain how to understand intuitively the monotonically decreasing behavior of $\widetilde{S}_{n}$. In contrast to the proof in the last subsection, the idea we will describe does not depend on the details of the JT gravity background and thus it can be generalized to the other gravity cases as well.

The replica index $n$ is sometimes identified as an analogue of the inverse temperature (see e.g. [41]). Here we will pursue this analogy. To do this, let us consider the change of variable from $E$ to $\mathcal{E}$ given by ${ }^{9}$

$$
e^{-\mathcal{E}}=A(E)=\frac{e^{-\beta E}}{\xi+E}
$$

Then we find

$$
Z_{n}=\int_{E_{0}}^{\infty} d E \rho_{0}(E) A(E)^{n}=\int_{\mathcal{E}_{0}}^{\infty} d \mathcal{E} D(\mathcal{E}) e^{-n \mathcal{E}},
$$

where $\mathcal{E}_{0}=-\log A\left(E_{0}\right)$ and

$$
D(\mathcal{E})=\left(\frac{\partial \mathcal{E}}{\partial E}\right)^{-1} \rho_{0}(E)=\frac{\xi+E}{\beta(\xi+E)+1} \rho_{0}(E) .
$$

$Z_{n}$ in (5.17) takes the form of the canonical partition function with inverse temperature $n$ and density of states $D(\mathcal{E})$. In this picture the "thermodynamic entropy" is expressed as $[41,42]$

$$
S_{\text {therm }}=\left(1-n \partial_{n}\right) \log Z_{n}
$$

On the other hand, as we saw in the last subsection $\partial_{t} \widetilde{S}_{n}<0$ is equivalent to (5.12), which is written as

$$
-\partial_{n} \frac{\partial_{t} Z_{n}}{n Z_{n}}=\frac{1}{n^{2}} \partial_{t} S_{\text {therm }}<0 .
$$

Therefore, the monotonically decreasing behavior of $\widetilde{S}_{n}$ is interpreted as that of the thermodynamic entropy $S_{\text {therm }}$.

\footnotetext{
${ }^{8}$ This is easily seen from the graph of $\sqrt{E} I_{1}(2 \sqrt{E})=\left(I_{1}(2 \sqrt{E}) / \sqrt{E}\right) \times E$ in figure 1 .

${ }^{9}(5.16)$ is rewritten as

$$
\beta(\xi+E) e^{\beta(\xi+E)}=\beta e^{\mathcal{E}+\beta \xi} .
$$
}

This is solved by the Lambert function $W(z) e^{W(z)}=z$ as

$$
\beta(\xi+E)=W\left(\beta e^{\mathcal{E}+\beta \xi}\right) .
$$




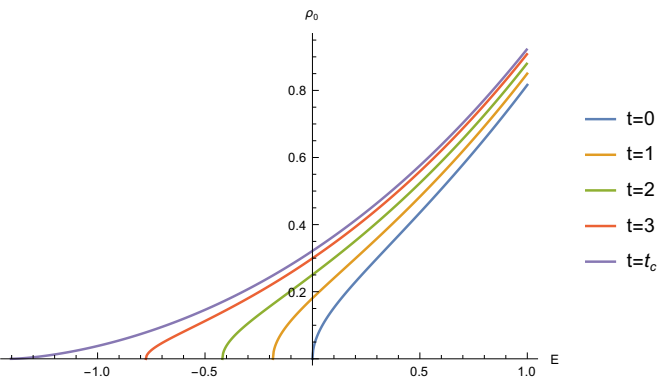

(a) $\rho_{0}(E)$ vs. $E$.

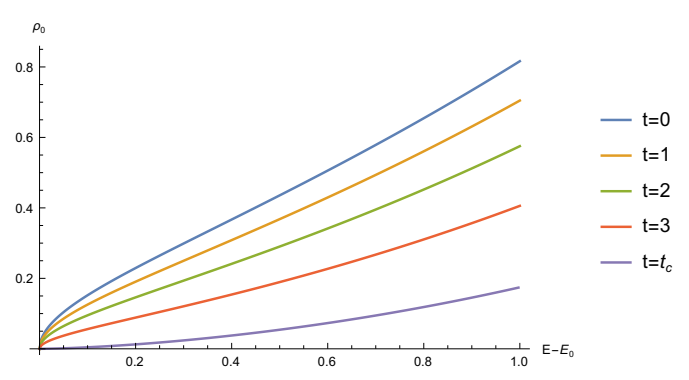

(b) $\rho_{0}(E)$ vs. $E-E_{0}$.

Figure 6. Plot of $\rho_{0}(E)$ against (a) $E$ and (b) $E-E_{0}$ for anti-FZZT branes. We set $\xi=18$ in this plot.

Let us list some useful properties of $S_{\text {therm }}$ :

1. The threshold energy $\mathcal{E}_{0}$ does not contribute to $S_{\text {therm }}$ : if we define $\widetilde{Z}_{n}$ by

$$
Z_{n}=e^{-n \mathcal{E}_{0}} \widetilde{Z}_{n}, \quad \widetilde{Z}_{n}=\int_{0}^{\infty} d \mathcal{E} D\left(\mathcal{E}+\mathcal{E}_{0}\right) e^{-n \mathcal{E}},
$$

then we find

$$
S_{\text {therm }}=\left(1-n \partial_{n}\right)\left(-n \mathcal{E}_{0}+\log \widetilde{Z}_{n}\right)=\left(1-n \partial_{n}\right) \log \widetilde{Z}_{n} .
$$

2. The overall scale of $\widetilde{Z}_{n}$ does contribute to $S_{\text {therm }}$ : if we define

$$
\widetilde{Z}_{n}=e^{S(t)} Z_{n}^{\prime}
$$

where $S(t)$ is $n$-independent, then we find

$$
S_{\text {therm }}=\left(1-n \partial_{n}\right)\left(S(t)+\log Z_{n}^{\prime}\right)=S(t)+\left(1-n \partial_{n}\right) \log Z_{n}^{\prime} .
$$

If we further assume that $Z_{n}^{\prime}$ is $t$-independent, then the monotonically decreasing behavior of $S(t)$ implies that of $S_{\text {therm }}$.

3. $S_{\text {therm }}$ is written as

$$
S_{\text {therm }}=\log \widetilde{Z}_{n}+n\langle\mathcal{E}\rangle,
$$

where $\langle\mathcal{E}\rangle$ is given by

$$
\langle\mathcal{E}\rangle=-\partial_{n} \log \widetilde{Z}_{n}=\frac{1}{\widetilde{Z}_{n}} \int_{0}^{\infty} d \mathcal{E} D\left(\mathcal{E}+\mathcal{E}_{0}\right) e^{-n \mathcal{E}} \mathcal{E}
$$

Let us now focus on the case of JT gravity with anti-FZZT branes. In figure 6 we plot $\rho_{0}(E)$ in (2.32) for several different values of $t$. Due to property 1 of $S_{\text {therm }}$, it is convenient to plot $\rho_{0}(E)$ against $E-E_{0}$ (see figure $6 \mathrm{~b}$ ) to consider the behavior of the entropy. Then we observe that the overall scale of $\rho_{0}$ clearly decreases as $t$ grows. ${ }^{10}$ By crude approximation,

\footnotetext{
${ }^{10}$ As we see in $(5.18), D(\mathcal{E})$ is proportional to $\rho_{0}(E)$ up to a prefactor. Since this prefactor is independent of $t$, the graph of $D(\mathcal{E})$ against $\mathcal{E}-\mathcal{E}_{0}$ also decreases as $t$ grows, in a similar way as $\rho_{0}(E)$ in figure $6 \mathrm{~b}$.
} 
the overall scale of $\rho_{0}$ gives that of $\widetilde{Z}_{n}$ and thus this implies the decreasing behavior of $S_{\text {therm }}$, as explained in property 2 . More precisely, as described in property $3, S_{\text {therm }}$ is related to $\widetilde{Z}_{n}$ by (5.25). We found numerically that each individual terms $\log \widetilde{Z}_{n}$ and $n\langle\mathcal{E}\rangle$ are not necessarily monotonically decreasing functions of $t$ for generic values of $\xi$, but the sum of them is always monotonically decreasing.

To summarize, we have seen that the monotonically decreasing behavior of $\widetilde{S}_{n}$ is equivalent to that of the "thermodynamic entropy" $S_{\text {therm }}$ if we regard the replica index $n$ as the inverse temperature. Its decreasing behavior is intuitively understood from that of the overall scale of $\rho_{0}(E)$.

It is well known that the entropy of the Hawking radiation is bounded from above by the thermodynamic entropy $S_{\mathrm{BH}}$ of the black hole, which is given by the area of horizon in the semi-classical approximation. For an evaporating black hole, the area of horizon decreases as time passes and this explains the decreasing behavior of the Page curve. As we saw in (4.7), $\widetilde{S}_{n}$ (not $S_{\text {therm }}$ ) corresponds to $S_{\mathrm{BH}}$ for $n>1$. In general, $\widetilde{S}_{n}$ and $S_{\text {therm }}$ are different quantities. However, one can easily see that $\widetilde{S}_{n}$ becomes equal to $S_{\text {therm }}$ in the limit $n \rightarrow 1$

$$
\lim _{n \rightarrow 1} \widetilde{S}_{n}=\lim _{n \rightarrow 1} S_{\text {therm }}
$$

Thus the thermodynamic entropy $S_{\mathrm{BH}}$ of black hole literally corresponds to the "thermodynamic entropy" $S_{\text {therm }}$ in the limit $n \rightarrow 1$.

\section{Dilaton gravity}

Recently, dilaton gravities with nontrivial dilaton potential were studied as deformations of JT gravity [28, 29]. Black hole solutions in these gravities were also discussed in [30]. JT gravity with (anti-)FZZT branes can be viewed as this type of dilaton gravity [24]. In this section we will study black hole solutions from the viewpoint of dilaton gravity. ${ }^{11}$

The action of dilaton gravity is written as [30]

$$
I=-\frac{1}{2} \int d^{2} x \sqrt{g}(\phi R+W(\phi))
$$

We derived that in the case of JT gravity with $K$ (anti-)FZZT branes the dilaton potential is given by $[24]$

$$
W(\phi)= \begin{cases}2 \phi+\frac{t \sqrt{2 \xi}}{\xi+\pi^{2} \phi^{2}} e^{-2 \pi \phi} & (\text { anti-FZZT }), \\ 2 \phi-\frac{t \sqrt{2 \xi}}{\xi+\pi^{2} \phi^{2}} e^{-2 \pi \phi} & (\mathrm{FZZT}) .\end{cases}
$$

The general Euclidean black hole solution is given by

$$
d s^{2}=A(r) d t^{2}+\frac{d r^{2}}{A(r)}, \quad \phi(r)=r, \quad A(r)=\int_{r_{h}}^{r} d \phi W(\phi),
$$

where $r=r_{h}$ is the horizon at which $A(r)$ vanishes. This is a one-parameter family of solutions parametrized by $r_{h}=\phi_{h}$. The value of $\phi_{h}$ is not fixed by the equation of motion.

\footnotetext{
${ }^{11}$ See also [43] for recent related studies.
} 


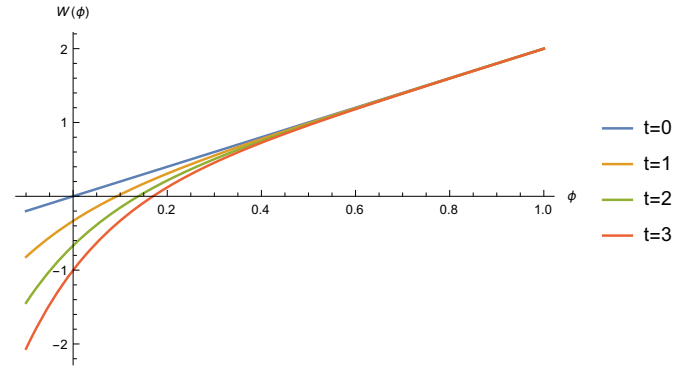

(a) $W(\phi)$ for FZZT branes.



(b) $W(\phi)$ for anti-FZZT branes.

Figure 7. Plot of $W(\phi)$ in (6.2) for (a) FZZT branes and (b) anti-FZZT branes. We set $\xi=18$ in this plot.

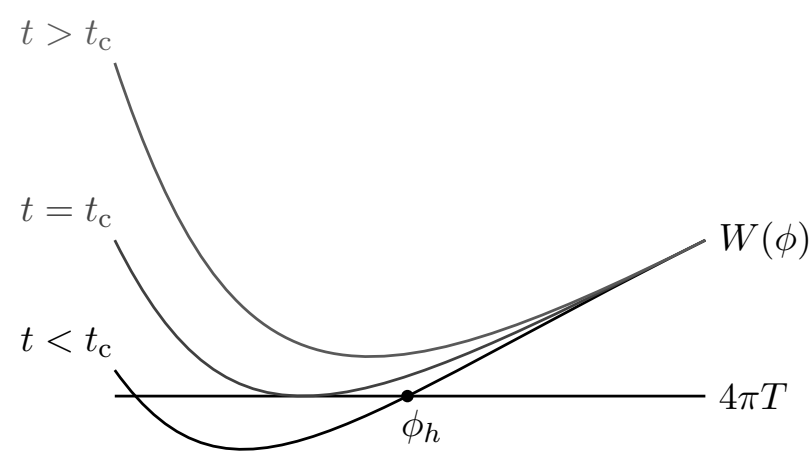

Figure 8. Stability of black hole solutions in the anti-FZZT brane setup. The largest root $\phi_{h}$ of (6.5) corresponds to the stable solution. The solution no longer exists for $t>t_{\mathrm{c}}$.

The entropy of this solution is given by

$$
S=2 \pi \phi_{h}+S_{0}
$$

The physical condition is $A(r)>0$ for $r>r_{h}$.

For a fixed temperature $T, \phi_{h}$ is determined by the condition

$$
\frac{W\left(\phi_{h}\right)}{4 \pi}=T .
$$

In figure 7 we show the plot of $W(\phi)$. For the FZZT branes in figure $7 \mathrm{a},(6.5)$ has a unique solution $\phi_{h}$ for a given value of $T$. As $t$ increases, $\phi_{h}$ also increases. Thus the entropy increases as a function of $t$.

On the other hand, from figure 7b for the anti-FZZT branes, one can see that there are two solutions of (6.5) if $t$ is not too large. As discussed in [30], the stable solution with minimal free energy corresponds to the largest root $\phi_{h}$ of (6.5) (see figure 8). The largest root $\phi_{h}$ of (6.5) decreases as $t$ increases. This explains the decreasing behavior of the entropy (6.4). Beyond some critical value $t=t_{\mathrm{c}}$, there is no solution of (6.5) for a given temperature. This might be interpreted that for $t>t_{\mathrm{c}}$ there is no stable black hole solutions; at $t=t_{\mathrm{c}}$ the stable black hole disappears. This suggests that, to model the black hole evaporation process, the anti-FZZT brane setup is more suitable than the FZZT brane setup. 


\section{Conclusion and outlook}

In this paper we studied the entanglement entropy in the matrix model of JT gravity with anti-FZZT branes, which serves as a toy model of an evaporating black hole. The entanglement entropy is defined between the color and flavor sectors, which correspond respectively to bulk gravity and to the interior partners of the early Hawking radiation. We computed the entropy in the planar approximation as well as in the 't Hooft limit. The 't Hooft coupling $t$, which is proportional to the number of branes, plays the role of time. We computed numerically the von Neumann and Rényi entropies as functions of $t$. In both cases, the entropy first increases and then decreases, which is peculiar to the Page curve of an evaporating black hole. We stress that we treated the anti-FZZT branes as dynamical objects and this was crucial to reproduce the late-time decreasing behavior of the entropy, because otherwise the entropy approaches a constant value at late time in the probe brane approximation [13], as we saw in figure 3 .

We saw that the system exhibits a phase transition at $t=t_{\mathrm{c}}$. This may be viewed as the end of the evaporation of the black hole. We studied the critical behavior of the entropy and derived that it scales as in (3.8). As $t$ grows toward $t=t_{\mathrm{c}}$, the Rényi entropy becomes dominated by $\widetilde{S}_{n}$ in (4.2). We conjectured that $\widetilde{S}_{n}$ monotonically decreases and proved this conjecture in the large $\xi$ limit. We also gave an intuitive explanation of this decreasing behavior. We studied black hole solutions of dilaton gravity that describes JT gravity with (anti-)FZZT branes and saw the continuous growth of the entropy in the FZZT setup as well as a phase transition in the anti-FZZT setup. This suggests that the anti-FZZT brane setup is more suitable to model an evaporating black hole.

There are many interesting open questions. We have seen that our model of dynamical branes in JT gravity serves as a good toy model for an evaporating black hole. We hope that the behavior of our model beyond the phase transition $t>t_{\mathrm{c}}$ would shed light on the deep question of the unitarity in black hole evaporation, e.g. the final state proposal in [44]. It would be interesting to study our matrix model beyond the phase transition $t>t_{\mathrm{c}}$ along the lines of [25]. Our analysis of the Page curve was limited to the planar approximation. It would be interesting to compute the higher genus corrections to the Page curve. More ambitiously, it would be very interesting if we can compute the Page curve of our model non-perturbatively in $g_{\mathrm{s}}$. We leave this as an interesting future problem. We can also repeat the analysis of the Petz map in [13] using our setup of dynamical branes. It would be interesting to study how the entanglement wedge reconstruction is modified from the result of [13] if we take account of the back-reaction of branes.

In our calculation of the Page curve, the decreasing behavior of entropy comes from the last term of (2.23), which is interpreted as a contribution of replica wormholes [13, 14]. The appearance of the replica wormhole is closely related to the ensemble average on the boundary side of the AdS/CFT correspondence. The role of the ensemble average in the gravitational path integral is still not well-understood and there are many conceptual issues related to this problem, such as the factorization puzzle (see e.g. [45-50] and references therein). It is believed that the Rényi entropy is a self-averaging quantity [13]. Nonetheless, it would be interesting to see how the Page curve of our model would look like if we pick a 
certain member of the ensemble and do not take an average over the random matrix (see e.g. [51] for a study in this direction).

\section{Acknowledgments}

This work was supported in part by JSPS KAKENHI Grant Nos. 19K03845, 19K03856, 21H05187 and JSPS Japan-Russia Research Cooperative Program. A preliminary result of this work was presented by one of the authors (KO) in the KMI colloquium at Nagoya University on October 13, 2021.

\section{A Schwinger-Dyson equation from saddle point approximation}

In this appendix we will derive the Schwinger-Dyson equation (2.22) based on the saddle point method.

Let us consider the integral

$$
\int \prod_{i=1}^{K} d \phi_{i}^{\dagger} d \phi_{i} e^{-\sum_{i j} \phi_{i}^{\dagger}\left(\lambda \delta_{i j}-\varrho_{i j}\right) \phi_{j}} .
$$

Then the two point function $\overline{\phi_{i} \phi_{j}^{\dagger}}$ is equal to the resolvent

$$
\overline{\phi_{i} \phi_{j}^{\dagger}}=(\lambda-\varrho)_{i j}^{-1}
$$

We can rewrite the integral as

$$
\begin{aligned}
\int d \phi^{\dagger} d \phi e^{-\phi^{\dagger}(\lambda-\varrho) \phi} & =\int d \phi^{\dagger} d \phi d G_{i j} \delta\left(G_{i j}-\phi_{i} \phi_{j}^{\dagger}\right) e^{-\operatorname{Tr}(\lambda-\varrho) G} \\
& =\int d \phi^{\dagger} d \phi d G d \Sigma e^{\Sigma_{j i}\left(G_{i j}-\phi_{i} \phi_{j}^{\dagger}\right)-\operatorname{Tr}(\lambda-\varrho) G} \\
& =\int d G d \Sigma e^{\operatorname{Tr} \Sigma G-\operatorname{Tr} \log \Sigma-\operatorname{Tr}(\lambda-\varrho) G}
\end{aligned}
$$

The density matrix $\varrho_{i j}$ is given by

$$
\varrho_{i j}=\frac{C_{i}^{\dagger} A C_{j}}{K Z_{1}}=C_{i}^{\dagger} \widehat{A} C_{j}, \quad \widehat{A}=\frac{A}{K Z_{1}} .
$$

After integrating out $C^{\dagger}, C$ we have

$$
\int d C^{\dagger} d C e^{-\operatorname{Tr} C^{\dagger} C+\operatorname{Tr} \varrho G}=e^{-\operatorname{Tr} \log (1-\widehat{A} G)},
$$

where $\operatorname{Tr} \log (1-\widehat{A} G)$ should be understood as the trace of both color and flavor indices. Then (A.3) becomes $\int d G d \Sigma e^{-I}$ where the action $I$ is given by

$$
I=-\operatorname{Tr} \Sigma G+\operatorname{Tr} \log \Sigma+\lambda \operatorname{Tr} G+\operatorname{Tr} \log (1-\widehat{A} G) .
$$


In the planar approximation, the $G$ - and $\Sigma$-integrals can be evaluated by the saddle point approximation. The saddle point equations read

$$
\begin{aligned}
\frac{\partial I}{\partial \Sigma_{i j}} & =-G_{i j}+\left(\Sigma^{-1}\right)_{i j}=0, \\
\frac{\partial I}{\partial G_{i j}} & =-\Sigma_{i j}+\lambda \delta_{i j}-\delta_{i j} \operatorname{Tr} \frac{\widehat{A}}{1-\widehat{A} G}=0 .
\end{aligned}
$$

Multiplying the second equation of (A.7) by $G_{i j}$ and summing over $i, j$, we find

$$
-K+\lambda \operatorname{Tr} G-\operatorname{Tr} G \operatorname{Tr} \frac{\widehat{A}}{1-\widehat{A} G}=0
$$

This is rewritten as

$$
\begin{aligned}
\lambda \operatorname{Tr} G & =K+\operatorname{Tr} G \operatorname{Tr} \frac{\widehat{A}}{1-\widehat{A} G} \\
& =K+\operatorname{Tr} G \sum_{n=1}^{\infty} \operatorname{Tr} \widehat{A}^{n} \operatorname{Tr} G^{n-1} .
\end{aligned}
$$

In the planar approximation we have

$$
\operatorname{Tr} G \approx \sum_{i} \overline{\phi_{i} \phi_{i}^{\dagger}}=\sum_{i}(\lambda-\varrho)_{i i}^{-1}=R(\lambda) .
$$

We also find

$$
\begin{aligned}
\operatorname{Tr} G^{n} & =\overline{\phi_{i_{1}} \phi_{i_{2}}^{\dagger} \phi_{i_{2}} \phi_{i_{3}}^{\dagger} \cdots \phi_{i_{n}} \phi_{i_{1}}^{\dagger}} \\
& \approx \overline{\phi_{i_{1}}^{\dagger} \phi_{i_{1}}} \cdot \overline{\phi_{i_{2}}^{\dagger} \phi_{i_{2}} \cdots \overline{\phi_{i_{n}}^{\dagger} \phi_{i_{n}}}} \\
& =(\operatorname{Tr} G)^{n}=R(\lambda)^{n} .
\end{aligned}
$$

Finally, (A.9) becomes

$$
\lambda R(\lambda)=K+\sum_{n=1}^{\infty} \operatorname{Tr} \widehat{A}^{n} R(\lambda)^{n} .
$$

Thus we have re-derived the Schwinger-Dyson equation (2.22), which was originally derived by means of diagrams in [13]. The above saddle point method can be generalized to the Grassmann-odd integral (i.e. to the case of FZZT branes).

Open Access. This article is distributed under the terms of the Creative Commons Attribution License (CC-BY 4.0), which permits any use, distribution and reproduction in any medium, provided the original author(s) and source are credited.

\section{References}

[1] S.W. Hawking, Breakdown of Predictability in Gravitational Collapse, Phys. Rev. D 14 (1976) 2460 [INSPIRE].

[2] S.W. Hawking, Particle Creation by Black Holes, Commun. Math. Phys. 43 (1975) 199 [Erratum ibid. 46 (1976) 206] [INSPIRE]. 
[3] D.N. Page, Information in black hole radiation, Phys. Rev. Lett. 71 (1993) 3743 [hep-th/9306083] [INSPIRE].

[4] G. Penington, Entanglement Wedge Reconstruction and the Information Paradox, JHEP 09 (2020) 002 [arXiv: 1905.08255] [inSPIRE].

[5] A. Almheiri, N. Engelhardt, D. Marolf and H. Maxfield, The entropy of bulk quantum fields and the entanglement wedge of an evaporating black hole, JHEP 12 (2019) 063 [arXiv: 1905.08762] [INSPIRE].

[6] A. Almheiri, R. Mahajan, J.M. Maldacena and Y. Zhao, The Page curve of Hawking radiation from semiclassical geometry, JHEP 03 (2020) 149 [arXiv:1908.10996] [INSPIRE].

[7] S. Ryu and T. Takayanagi, Holographic derivation of entanglement entropy from AdS/CFT, Phys. Rev. Lett. 96 (2006) 181602 [hep-th/0603001] [INSPIRE].

[8] V.E. Hubeny, M. Rangamani and T. Takayanagi, A Covariant holographic entanglement entropy proposal, JHEP 07 (2007) 062 [arXiv:0705.0016] [INSPIRE].

[9] A. Lewkowycz and J.M. Maldacena, Generalized gravitational entropy, JHEP 08 (2013) 090 [arXiv: 1304.4926] [INSPIRE].

[10] T. Barrella, X. Dong, S.A. Hartnoll and V.L. Martin, Holographic entanglement beyond classical gravity, JHEP 09 (2013) 109 [arXiv: 1306.4682] [INSPIRE].

[11] T. Faulkner, A. Lewkowycz and J.M. Maldacena, Quantum corrections to holographic entanglement entropy, JHEP 11 (2013) 074 [arXiv: 1307.2892] [INSPIRE].

[12] N. Engelhardt and A.C. Wall, Quantum Extremal Surfaces: Holographic Entanglement Entropy beyond the Classical Regime, JHEP 01 (2015) 073 [arXiv:1408.3203] [INSPIRE].

[13] G. Penington, S.H. Shenker, D. Stanford and Z. Yang, Replica wormholes and the black hole interior, arXiv: 1911.11977 [INSPIRE].

[14] A. Almheiri, T. Hartman, J.M. Maldacena, E. Shaghoulian and A. Tajdini, Replica Wormholes and the Entropy of Hawking Radiation, JHEP 05 (2020) 013 [arXiv: 1911.12333] [INSPIRE].

[15] A. Almheiri, T. Hartman, J.M. Maldacena, E. Shaghoulian and A. Tajdini, The entropy of Hawking radiation, Rev. Mod. Phys. 93 (2021) 035002 [arXiv: 2006.06872] [INSPIRE].

[16] R. Jackiw, Lower Dimensional Gravity, Nucl. Phys. B 252 (1985) 343 [InSPIRE].

[17] C. Teitelboim, Gravitation and Hamiltonian Structure in Two Space-Time Dimensions, Phys. Lett. B 126 (1983) 41 [InSPIRE].

[18] A. Goel, L.V. Iliesiu, J. Kruthoff and Z. Yang, Classifying boundary conditions in JT gravity: from energy-branes to $\alpha$-branes, JHEP 04 (2021) 069 [arXiv:2010.12592] [INSPIRE].

[19] D.N. Page, Average entropy of a subsystem, Phys. Rev. Lett. 71 (1993) 1291 [gr-qc/9305007] [INSPIRE].

[20] K. Okuyama, Capacity of entanglement in random pure state, Phys. Lett. B $\mathbf{8 2 0}$ (2021) 136600 [arXiv: 2103.08909] [INSPIRE].

[21] P. Saad, S.H. Shenker and D. Stanford, JT gravity as a matrix integral, arXiv:1903.11115 [INSPIRE].

[22] V. Fateev, A.B. Zamolodchikov and A.B. Zamolodchikov, Boundary Liouville field theory. 1. Boundary state and boundary two point function, hep-th/0001012 [INSPIRE]. 
[23] J. Teschner, Remarks on Liouville theory with boundary, PoS tmr2000 (2000) 041 [hep-th/0009138] [INSPIRE].

[24] K. Okuyama and K. Sakai, FZZT branes in JT gravity and topological gravity, JHEP 09 (2021) 191 [arXiv : 2108.03876] [inSPIRE].

[25] P. Gao, D.L. Jafferis and D.K. Kolchmeyer, An effective matrix model for dynamical end of the world branes in Jackiw-Teitelboim gravity, JHEP 01 (2022) 038 [arXiv:2104.01184] [INSPIRE].

[26] K. Goto, T. Hartman and A. Tajdini, Replica wormholes for an evaporating $2 D$ black hole, JHEP 04 (2021) 289 [arXiv:2011.09043] [INSPIRE].

[27] M. Cadoni and A.P. Sanna, Unitarity and Page curve for evaporation of $2 D$ AdS black holes, $\operatorname{arXiv}: 2106.14738$ [INSPIRE].

[28] H. Maxfield and G.J. Turiaci, The path integral of 3D gravity near extremality; or, JT gravity with defects as a matrix integral, JHEP 01 (2021) 118 [arXiv:2006.11317] [INSPIRE].

[29] E. Witten, Matrix Models and Deformations of JT Gravity, Proc. Roy. Soc. Lond. A 476 (2020) 20200582 [arXiv:2006.13414] [INSPIRE].

[30] E. Witten, Deformations of JT Gravity and Phase Transitions, arXiv:2006.03494 [INSPIRE].

[31] S. Sugiura and A. Shimizu, Canonical Thermal Pure Quantum State, Phys. Rev. Lett. 111 (2013) 010401 [arXiv: 1302.3138] [INSPIRE].

[32] K. Goto, Y. Kusuki, K. Tamaoka and T. Ugajin, Product of random states and spatial (half-)wormholes, JHEP 10 (2021) 205 [arXiv:2108.08308] [InSPIRE].

[33] M. Mulase and B. Safnuk, Mirzakhani's recursion relations, Virasoro constraints and the KdV hierarchy, math/0601194 [INSPIRE].

[34] R. Dijkgraaf and E. Witten, Developments in Topological Gravity, Int. J. Mod. Phys. A 33 (2018) 1830029 [arXiv: 1804.03275] [inSPIRE].

[35] K. Okuyama and K. Sakai, JT gravity, KdV equations and macroscopic loop operators, JHEP 01 (2020) 156 [arXiv:1911.01659] [INSPIRE].

[36] N. Seiberg and D. Starnford, unpublished.

[37] S. Hirano and T. Kuroki, Replica wormholes from Liouville theory, JHEP 01 (2022) 094 [arXiv:2109.12539] [INSPIRE].

[38] E. Date, M. Jimbo, M. Kashiwara and T. Miwa, Transformation groups for soliton equations - Euclidean Lie algebras and reduction of the KP hierarchy, Publ. Res. Inst. Math. Sci. Kyoto 18 (1982) 1077.

[39] C.V. Johnson and F. Rosso, Solving Puzzles in Deformed JT Gravity: Phase Transitions and Non-Perturbative Effects, JHEP 04 (2021) 030 [arXiv: 2011.06026] [InSPIRE].

[40] C. Itzykson and J.B. Zuber, Combinatorics of the modular group. 2. The Kontsevich integrals, Int. J. Mod. Phys. A 7 (1992) 5661 [hep-th/9201001] [INSPIRE].

[41] Y. Nakaguchi and T. Nishioka, A holographic proof of Rényi entropic inequalities, JHEP 12 (2016) 129 [arXiv: 1606. 08443] [INSPIRE].

[42] X. Dong, The Gravity Dual of Renyi Entropy, Nature Commun. 7 (2016) 12472 [arXiv: 1601.06788] [INSPIRE]. 
[43] P. Gregori and R. Schiappa, From Minimal Strings towards Jackiw-Teitelboim Gravity: On their Resurgence, Resonance, and Black Holes, arXiv:2108.11409 [INSPIRE].

[44] G.T. Horowitz and J.M. Maldacena, The Black hole final state, JHEP 02 (2004) 008 [hep-th/0310281] [INSPIRE].

[45] D. Marolf and H. Maxfield, Transcending the ensemble: baby universes, spacetime wormholes, and the order and disorder of black hole information, JHEP 08 (2020) 044 [arXiv: 2002.08950] [INSPIRE].

[46] J. McNamara and C. Vafa, Baby Universes, Holography, and the Swampland, arXiv:2004.06738 [INSPIRE].

[47] P. Saad, S.H. Shenker, D. Stanford and S. Yao, Wormholes without averaging, arXiv:2103.16754 [INSPIRE].

[48] P. Saad, S. Shenker and S. Yao, Comments on wormholes and factorization, arXiv:2107.13130 [INSPIRE].

[49] A. Blommaert, L.V. Iliesiu and J. Kruthoff, Gravity factorized, arXiv:2111.07863 [INSPIRE].

[50] J.J. Heckman, A.P. Turner and X. Yu, Disorder Averaging and its UV (Dis)Contents, arXiv:2111.06404 [INSPIRE].

[51] A. Blommaert and M. Usatyuk, Microstructure in matrix elements, arXiv:2108.02210 [INSPIRE]. 